\title{
An Empirical Evaluation of Common Vector Based Classification Methods and Some Extensions ${ }^{\star}$
}

\author{
Katerine Díaz-Chito, Francesc J. Ferri, and Wladimiro Díaz-Villanueva \\ Dept. Informàtica, Universitat de València. Spain \\ \{Katerine.Diaz,Francesc.Ferri, Wladimiro.Diaz\}@uv.es
}

\begin{abstract}
An empirical evaluation of linear and kernel common vector based approaches has been considered in this work. Both versions are extended by considering directions (attributes) that carry out very little information as if they were null. Experiments on different kinds of data confirm that using this as a regularization parameter leads to usually better (and never worse) results than the basic algorithms.
\end{abstract}

\section{Introduction}

Recent developments in pattern recognition methodology suggest that good trade-offs between intrinsic complexity of the problems and models used to represent them constitute one of the key factors that drive the good behavior of some approaches against others [517. In particular, learning techniques that explicitly aim at reducing the complexity of the models either by reducing features, prototypes or both have experienced a deep development in the last decades 812 . Feature selection and extraction methods and dimensionality reduction in general are commonly used when there is enough a priori evidence that significant redundancy is present in the representation of data. Techniques that deal with high dimensional data by explicitly managing projections onto low dimensional subspaces are usually known as subspace methods [12]. In fact, many classical approaches to representation or classification such as principal component analysis (PCA) or Fisher or Linear Discriminant Analysis (LDA) can be properly included in this category.

The key idea of subspace methods consists of finding an appropriate subspace (and the corresponding projection operator) in which the original recognition problem becomes significantly easier or even trivial. Subspace methods in general have become an active field of research in recent times both because of the proliferation of practical problems exhibiting very high dimensional representations and also since the use of the kernel trick [15] has lead to a relatively easy way of extending the methods to the nonlinear case.

Subspace methods usually rely on the fact that the intrinsic dimensionality of the problem is much lower than the real dimension of the data. Moreover, usually

\footnotetext{
* This work has been partially funded by FEDER and Spanish MEC through projects DPI2006-15542-C04-04 and Consolider Ingenio 2010 CSD2007-00018.
} 
linear separability and dealing with relatively clean and well-behaved data (from a numerical stability point of view) needs to be assumed for its practical use. This is the case of appearance-based face recognition in which subspace methods have become increasingly popular [18].

Subspace methods based on the concept of common vectors have been recently proposed for classification problems in high dimensional spaces like speech and face recognition 64. Common vector methods can be thought of as projection methods that make all samples of a particular class to collapse on a single point (the common vector). In this subspace, the classification of test samples can be based on their (usually Euclidean) distance to the common vector in the appropriate subspace. Recent works have proposed extensions of the basic methods aimed at making them applicable to broader domains 317.

This paper presents an empirical evaluation of common vector based methods and their extensions in a range of different situations ranging from face recognition to graph-based chemical compound analysis. Further extensions and alternatives are introduced and studied in this particular context.

\section{The Discriminant Common Vector Method}

Let us suppose we have given $m$ samples in $\mathbb{R}^{n}$ (with $m<n$ ) where $m_{k}$ of them correspond to the $k$-th class, $\left\{x_{i}^{k}\right\}_{i=1}^{m_{k}}, \sum_{k} m_{k}=m$ and $k=1, \ldots, c$.

Let us define the mean vector for each class and the within and between class scatter matrices, $S_{W}$ and $S_{B}$ in the usual way $[5$. We use $R(S)$ and $N(S)$ to refer to the complementary range and null spaces of a scatter matrix $S$. For example, $R\left(S_{W}\right)$ and $N\left(S_{W}\right)$ are the linear subspaces spanned by the eigenvectors of $S_{W}$ corresponding to nonzero and zero eigenvalues, respectively.

It has been proved [4] that under certain assumptions all the samples from a particular class collapse into a unique vector when orthogonally projected onto $N\left(S_{W}\right)$. This consequently implies that the rank of the projected total scatter matrix, $S_{T}=S_{W}+S_{B}$ is $c-1$ (or less if two or more classes collapse together). If the $c$ common vectors are distinct, a projection onto a $c-1$ dimensional linear subspace exists that maps all training data into these vectors. New data can be then classified by projecting it into the same subspace and measuring the (usually Euclidean) distance to the common vectors of each class. This is a particular case of a nearest mean (NM) classifier and we will use this terminology in the present work. This method is known as the Discriminative Common Vector (DCV) approach and has been proposed in the context of very high dimensional data as in appearance-based face recognition [4] being the limitation of having more dimensions than data the most serious one to make the method broadly applicable.

The DCV method can be extended to the nonlinear case by applying the kernel trick 143. That is, by first projecting the data into a much higher, possibly infinite dimensional space implicitly using a given mapping, $\phi$. First, the data is projected onto the range space $R\left(S_{T}^{\phi}\right)=R\left(S_{W}^{\phi}+S_{B}^{\phi}\right)$ where the superscript indicates the new representation space. As in this new space, scatter matrices 
can be analytically obtained in terms of kernel matrices (given by dot products among given data), then the final projection onto $N\left(S_{W}^{\phi}\right)$ can be obtained also in terms of kernel matrices. A further PCA step is applied to obtain a final projection onto a $c-1$ subspace as in the case of linear DCV. In the kernel DCV $(\mathrm{kDCV})$ method, the distinctness of common vectors is guaranteed if the training kernel matrix is positive definite [3].

This nonlinear extension of the plain DCV has several practical advantages. First, more complex problems involving nonlinear dependences can be conveniently tackled. Also, the dependence of the cost on the number of dimensions is changed into dependence on the number of training samples. One practical drawback is that kernel parameters need to be tuned on the particular data. Also, care must be taken when applying kDCV because of overtraining that can appear more often than in the linear case.

\section{Extended Discriminant Common Vectors}

The Rough Common Vector method (rDCV) is a recent linear extension of the DCV method that has been proposed by relaxing the condition about the null space of $S_{W}$ [17. This consists basically in taking as zero the small enough eigenvalues from the eigendecomposition of $S_{W}$. This on one hand makes not possible the use of the differencing methods proposed in [4] but instead overcomes the limitation of $m<n$ and increases in some way the generalization ability of the method which is now controlled by the amount of extra dimensions which are included in the extended null space. The generalization ability of linear common vector based methods and how this behaves across different kinds of data has been studied only recently [1] and only from an empirical point of view.

In this paper, the same extension is included in the kDCV by setting a variance parameter, $f$, that controls the nonzero eigenvalues of $S_{W}^{\phi}$ that are taken as zero. In particular, if $\lambda_{i}$ are the normalized and increasingly ordered eigenvalues, we choose as the extended null space the subspace spanned by the eigenvectors corresponding to eigenvalues $\lambda_{1}, \ldots, \lambda_{s}$ satisfying $\sum_{i=1}^{s} \lambda_{i} \leq f$. Note that this parameter is different from the one introduced in [17] but in this way the parameter has the meaning of suppressed variance and we have the original DCV and $\mathrm{kDCV}$ methods when $f=0$ in both cases.

The effect of increasing $f$ in both linear and kernel DCV methods has not been fully studied. The observable effect is that the classes do not collapse anymore into common vectors. In [17] the projected mean of each class is taken as common vector and the $(c-1)$-dimensional subspace spanned by them as the final projection where distances to common vectors (i.e. NM) is used to classify.

But in fact, as classes do not collapse, the rank of $S_{T}$ or $S_{T}^{\phi}$ will be certainly greater than $c-1$ thus opening the possibility of considering final projected spaces of higher dimensions. Also and independently, the possibility of using all training data to classify instead of only common vectors can be considered by substituting the NM by the nearest(s) neighbor(s) (NN). These two possibilities are explored in this work using data from different domains. 

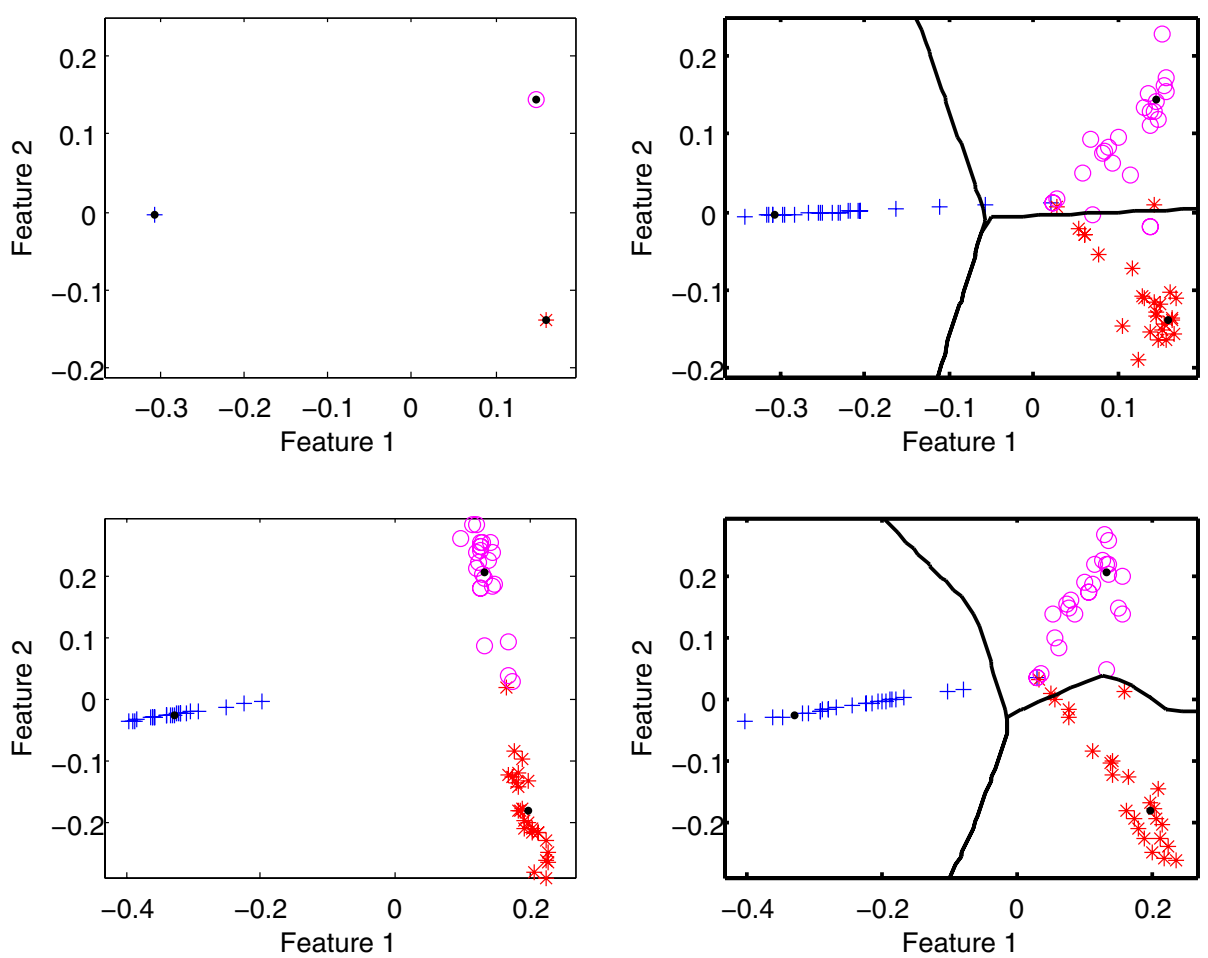

Fig. 1. Illustrative example of the Kernel DCV method and extension using the Iris database. The original method is used in the upper row and a variance parameter of 0.2 has been used for the lower row. Left column shows the projected training set and right column shows the projected test set along with the boundaries of the NM (upper) and NN (lower) classifiers.

Figure 1 contains an illustrative example of using $\mathrm{kDCV}$ on a random partition of the well-known Iris data into train and test sets. In the upper row, train (left) and test (right) sets are projected onto the final 2-dimensional space. Boundaries of the NM classifier are also shown along with the projected test set. In the lower row, $\mathrm{kDCV}$ with variance parameter of 0.2 is applied to the same data. NN boundaries are now shown instead. Although this is in fact a toy and very small example, it can be seen that the projected training set carries out significant information about how the (independent) test set will be projected onto the final space.

\section{Experimental Results}

A number of experiments have been conducted on several kinds of data of relatively high dimensionality in order to compare both linear and kernel versions of 


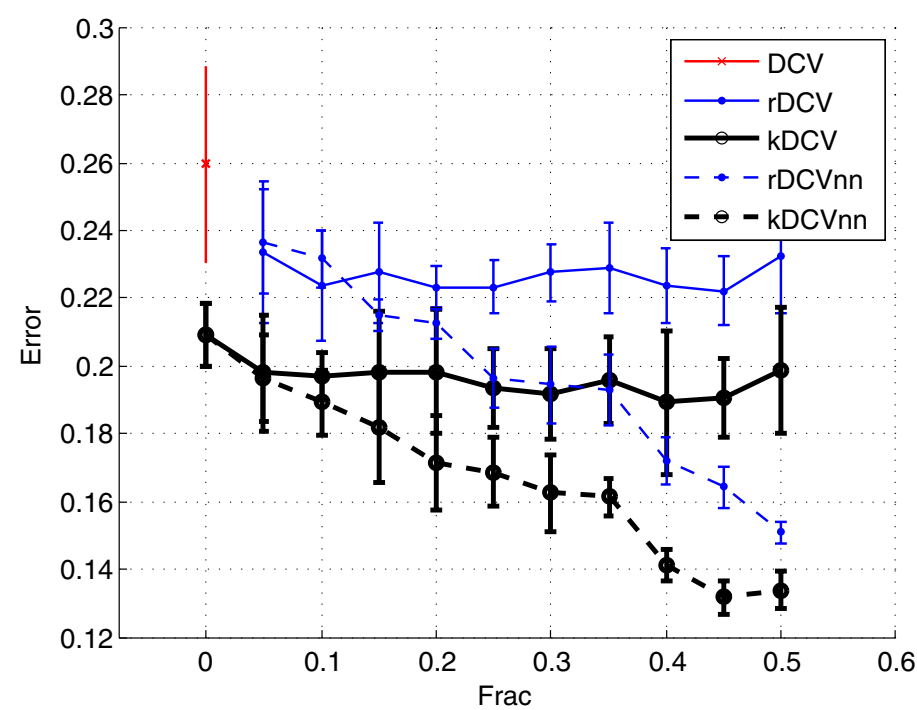

Fig. 2. Classification results using NM and NN classifiers on Coil dataset projected using DCV, rDCV, and $\mathrm{kDCV}$ using several values of the variance parameter $f$ (Frac). The kernel parameter for this data is 1000 .

the Discriminant Common Vector approach and their extensions using a variance parameter.

In all experiments using kernels, the Gaussian kernel with an appropriately tuned parameter has been considered. Values of the variance parameter ranging from zero (plain $\mathrm{kDCV}$ and $\mathrm{DCV}$ ) to 0.5 (half the total variance of the range space of $S_{W}^{\phi}$ or $\left.S_{W}\right)$ have been considered.

The first dataset considered is made from the Coil-100 dataset [11] by taking a random subset of 30 images of each of 40 different objects. Original images have been downsampled to 40x40. The final dataset consists then of $12001600-$ dimensional objects from 40 different classes.

The results of using the considered methods on this data are shown in Figure 2. As can be seen, kDCV methods significantly improve the plain DCV results. Also, the consideration of the variance parameter improves the results with regard to the plain methods. But this improvement is really significant when NN classifier is used in the projected space instead of the NM classifier.

In this experiment $1 / 3$ of each class is used for training and the remaining ones for test. Results are the average of 3 different and independent runs.

Given the nature of this dataset in which some of the objects are very different, the value of the variance parameter can be increased without producing high degrees of overlapping in the projected space.

The second dataset corresponds to a particular problem in the domain of classification of chemical compounds from their topological and structural description [10. In particular, a dataset of 434 samples consisting of 62 molecular 


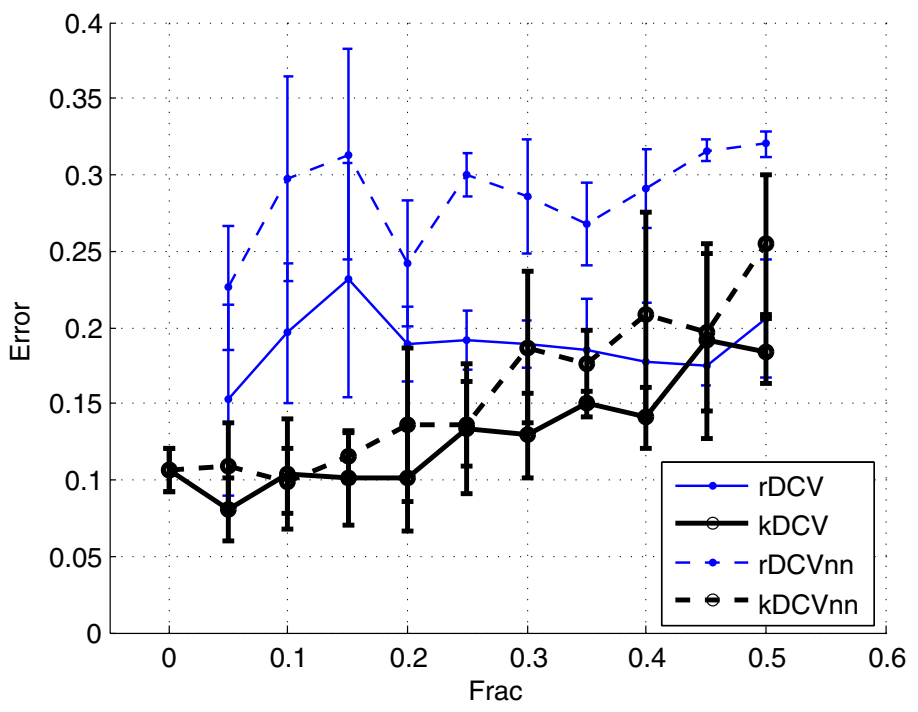

Fig. 3. Classification results using NM and NN classifiers on Antibacterial drug dataset projected using $\mathrm{rDCV}$, and $\mathrm{kDCV}$ using several values of the variance parameter $f$. The kernel parameter for this data is 10 .

descriptors computed from the graph corresponding to its chemical formula is considered. Roughly half of these compounds (218) are known to exhibit antibacterial activity. So the dataset consists of 434 62-dimensional objects from 2 classes. Note that in this case the plain DCV method cannot be used.

Experiments use $2 / 3$ of the available data for training and the remaining $1 / 3$ for testing in a classic 3 -fold cross validation scheme. The final results are the corresponding average that takes into account all data.

The results using this data are shown in Figure 3. In this case, no improvement is observed when the variance parameter is increased. Also, using NN instead of NM is always equal or worse. At least, it can be seen that $\mathrm{kDCV}$ with variance values from 0 to 0.2 behaves equally well and constitutes the best option for this data.

The last experiment involves data from frontal face images taken from the well-know datasets AR [9], ORL [13] and YALE [16]. All images have been manually aligned and conveniently cropped and downsampled to 40x40. Moreover, to study the behavior of the methods considered when data is degraded with noise, corrupted versions of the faces using variable amounts of Gaussian additive noise have been added. A total of 95 different classes is considered and there are 50 different images of each person. Training sets of 10 random images per person have been considered and results on the remaining 40 are averaged over 5 different runs.

Figure 4 shows the results obtained using both linear and nonlinear methods considered. In this case, plain DCV gives a significantly worse result. Both linear and kernel versions using NM exhibit a similar behavior with the variance 


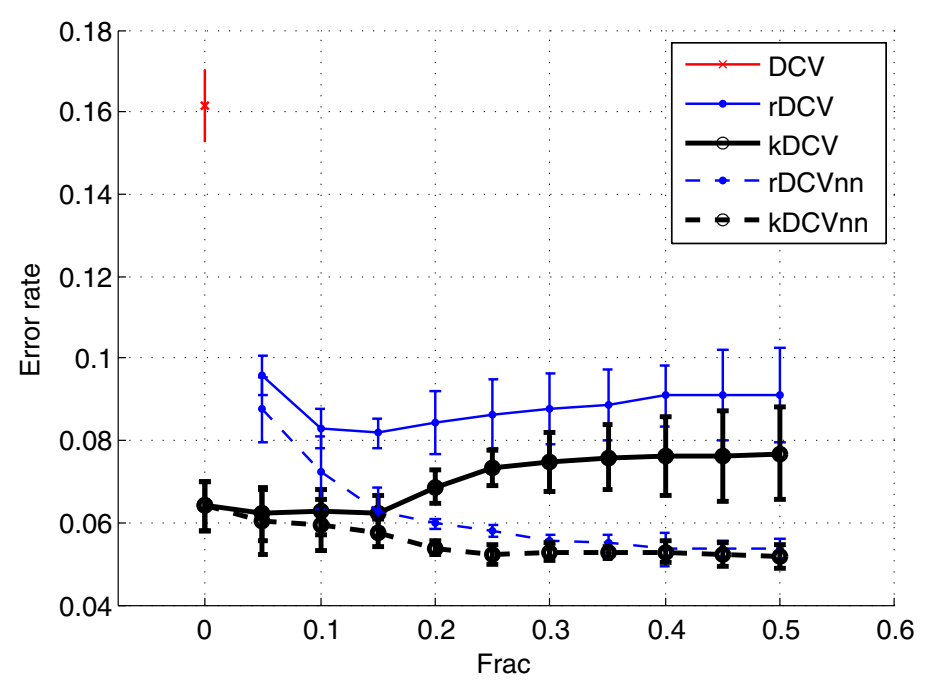

Fig. 4. Classification results using NM and NN classifiers on AR dataset (with Gaussian noise added) projected using $\mathrm{rDCV}$, and $\mathrm{kDCV}$ using several values of the variance parameter $f$. The kernel parameter for this data is 100 .

parameter. First a slight improvement or stability and then a slow decrease of performance from values of about 0.2 . On the other hand, when using NN both linear and kernel methods are able to significantly improve the results obtained for relatively high values of the variance parameter.

\section{Concluding Remarks and Further Work}

An empirical evaluation of linear and nonlinear common vector based classification approaches has been carried out on several different data domains. The methods have been extended by considering pseudo null spaces in which ortogonal directions that are discarded by plain DCV and kDCV are taken into account if their corresponding variance is small enough.

The main conclusion is that usually the kernel version of DCV improves the results of the linear case if the kernel parameter is appropriately tuned.

More importantly, from the results shown, the variance parameter can be used in most cases as a convenient regularization which leads to a better generalization abilities. In particular, using the NN classification rule using the projected training prototypes looks as a significantly better option that largely improves both DCV and kDCV without extensions.

Although experiments in this paper give results whose absolute values can be far from the state of the art in their corresponding domain (i.e. there are better adhoc solutions possibly using more apriori knowledge about the problem), their 
relative values allow us to draw conclusions about the relative merits of the different options.

More realistic experimentation in a more restricted domain in which the generalization ability is a key factor should be considered and is now under investigation. Further mechanisms to construct robust classifiers in the finally projected space could also be considered together with alternative and more effective ways of controlling generalization in these models.

\section{References}

1. Armengot, M., Ferri, F.J., Diaz-Villanueva, W.: Experiments about the generalization ability of common vector based methods for face recognition. In: Proceedings of PRIS 2007, Madeira, Portugal, ICEIS, pp. 129-137 (August 2007)

2. Bezdek, J.C., Kuncheva, L.: Some notes on twenty one (21) nearest prototype classifiers. In: Proceedings of the Joint IAPR International Workshops on Advances in Pattern Recognition, pp. 1-16. Springer, London (2000)

3. Cevikalp, H., Neamtu, M., Wilkes, M.: Discriminative common vector method with kernels. IEEE Transactions on Neural Networks 17(6), 1550-1565 (2006)

4. Cevikalp, H., Neamtu, M., Wilkes, M., Barkana, A.: Discriminative common vectors for face recognition. IEEE Transactions on Pattern Analysis and Machine Intelligence 27(1), 4-13 (2005)

5. Duda, R.O., Hart, P.E., Stork, D.G.: Pattern Classification, 2nd edn. John Wiley and Sons, Chichester (2001)

6. Gulmezoglu, M.B., Dzhafarov, V., Keskin, M., Barkana, A.: A novel approach to isolated word recognition. IEEE Transactions on Speech and Audio Processing 7(6), 620-628 (1999)

7. Jain, A.K., Duin, R.P.W., Mao, J.: Duin, and Jianchang Mao. Statistical pattern recognition: A review. IEEE Trans. Pattern Anal. Mach. Intell. 22(1), 4-37 (2000)

8. Liu, H., Motoda, H.: Feature Extraction, Construction and Selection: A Data Mining Perspective. Kluwer Academic Publishers, Norwell (1998)

9. Martinez, A.M., Benavente, R.: The AR face database. Technical Report 24, Computer Vision Center, Barcelona (1998)

10. Murcia-Soler, M., Pérez-Giménez, F., García-March, F.J., Salabert-Salvador, M.T., Díaz-Villanueva, W., Castro-Bleda, M.J., Villanueva-Pareja, A.: Artificial neural networks and linear discriminant analysis: A valuable combination in the selection of new antibacterial compounds. J. Chem. Inf. and Comp. Sciences 3, 1031-1041 (2004)

11. Nene, S.A., Nayar, S.K., Murase, H.: Columbia object image library (coil-100). Technical Report CUCS-006-96, Columbia University (February 1996)

12. Oja, E.: Subspace Methods of Pattern Recognition. Research Studies Press (1983)

13. Samaria, F., Harter, A.: Parameterisation of a stochastic model for human face identification. In: 2nd IEEE Workshop on Applications of Computer Vision (1994)

14. Scholkopf, B., Smola, A.: Learning with Kernels. MIT Press, Cambridge (2002)

15. Schölkopf, B., Smola, A., Müller, K.-R.: Nonlinear component analysis as a kernel eigenvalue problem. Neural Comput. 10(5), 1299-1319 (1998) 
16. Swets, D.L., Weng, J.: Using discriminant eigenfeatures for image retrieval. IEEE Trans. Pattern Anal. Mach. Intell. 18(8), 831-836 (1996)

17. Tamura, A., Zhao, Q.F.: Rough common vector: A new approach to face recognition. In: Proceedings of the 2007 SMC. Intl. Conf. on Syst., Man and Cyb., Montreal, Canada, pp. 2366-2371 (October 2007)

18. Turk, M., Pentland, A.: Eigenfaces for recognition. Journal of Cognitive Neuroscience 3(1), 71-86 (1991) 